\title{
Fractures among children: incidence and impact on daily activities
}

\author{
Branko Kopjar, Thomas M Wickizer
}

\begin{abstract}
Objectives-The study's objective was to examine incidence of fractures and associated activity restriction among children aged $0-12$ years.

Design-Injuries were prospectively recorded over the four year period from 1992-95 in a cohort of children aged 0-12 years, representing 193540 children years. Information about length and extent of activity restriction was collected from parents by a mailed questionnaire for a subsample of 192 children with a fracture.

Results-A total of 2477 fractures occurred in the study population (128 per 10000 children annually). The incidence increased linearly with age, by 14 cases per 10000 children year for each year of age. Boys and girls showed similar patterns of fracture occurrence. There was a significant difference in length of activity restrictions for different types of fractures. The mean and $95 \%$ confidence interval (CI) of activity restricted days for leg fractures were $26(95 \%$ CI 7 to 45$)$ days, for arm fractures, 14 (95\% CI 8 to 20) days, and for other fractures, 5 (95\% CI 1 to 8$)$ days. Arm fractures represented $66 \%$ of the cases and $62 \%$ of the activity restricted days; leg fractures $19 \%$ of cases and $33 \%$ of all activity restricted days; and other fractures $16 \%$ of the cases but only $5 \%$ of the activity restricted days in this population. Conclusion-The incidence of fractures increases in childhood. Different types of fractures among children cause different amounts of activity restriction.

(Injury Prevention 1998;4:194-197)
\end{abstract}

Keywords: fracture; activity restriction

Injuries are common among children, with an annual incidence of approximately $25 \% .{ }^{1-4} \mathrm{~A}$ majority of childhood injuries are minor events, but an unknown portion is a source of significant disability and morbidity. ${ }^{15}$ It is instinctively appealing to target injury preventive efforts towards more severe injuries, but, apart from those with a fatal outcome, little empirical information is available about consequences. Based on common sense and clinical experience, it has been suggested that burns, poisoning with lethal substances, brain injury with loss of consciousness, near drowning, and fractures represent the most severe injuries among children. ${ }^{5}$

Fractures are a common and significant injury in childhood, but the information about the pattern of fractures among children is scarce. Some information is available from studies that describe occurrence of childhood injuries in general, ${ }^{13}$ but little is known about the causes and types of fractures in different age groups, or about differences in occurrence between boys and girls. Fractures often result in temporary activity limitation, hospitalization, and sometimes in permanent disability. ${ }^{167}$ What has not been reported is the length of fracture related activity limitations. This information is important to better understand the consequences and may help in the creation of injury prevention policies. ${ }^{56}$

This study reports incidence data on fractures in a large Norwegian child population and analyzes the length of activity limitation due to different types of fractures.

\section{Methods}

CASES

The study population consisted of children aged 0-12 years, who were resident in 15 communities in the county of Rogaland located in southwestern Norway, over the period 199295. The average annual population less than 13 years during the study period was 48385 . A total of 193540 children years was represented in the study. The communities included in the study varied in size. The largest was Stavanger, a town of approximately 100000 and the smallest was Kvitsøy, with approximately 500 inhabitants.

Cases of fractures were identified through ongoing prospective registration of all injuries (regardless of age or diagnosis) performed by the Central Hospital in Stavanger. This hospital provides inpatient and outpatient medical care for fractures for the population in the study. No other medical facilities that treat fractures exist in these communities. Information about the injury event was collected at the time the injured child sought medical help. This information was obtained from the child if possible, or from someone accompanying him or her to the hospital.

The following information was obtained: age and sex, diagnosis, place where the injury occurred, activity at the time of the injury, mechanism of injury, and treatment disposition. Information about place and activity was used to classify events into four categories: traffic, school, sports, and other. Traffic included all events in which a motor vehicle, bicycle, or some other mode of land transport was used. School injuries were those occurring in schools, including gym classes and other school based sports activities. Sports injuries were all fractures occurring during organized 


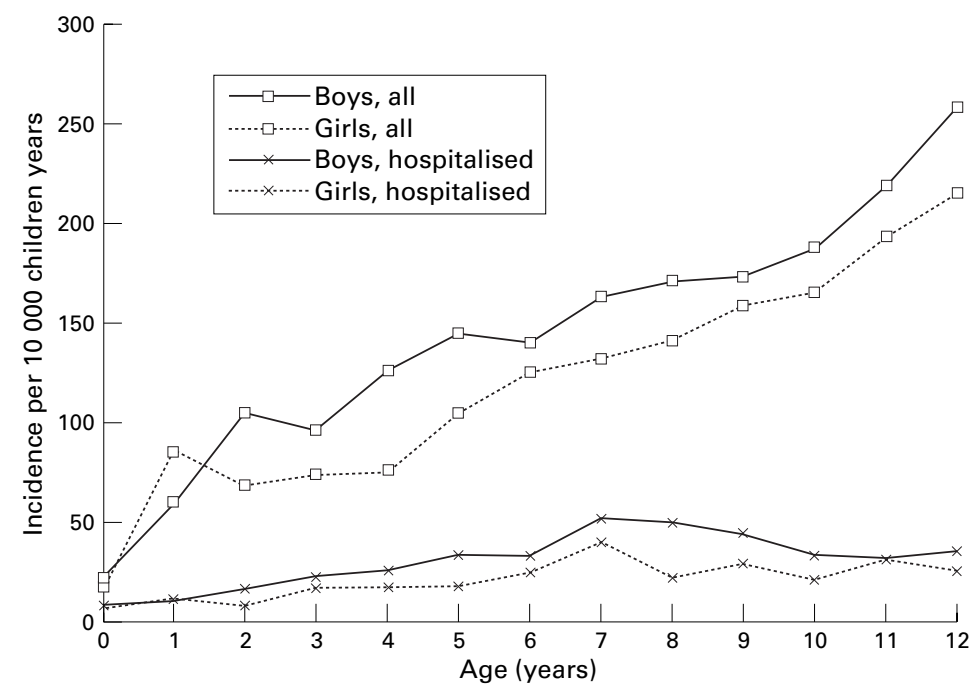

Figure 1 Annual incidence per 10000 children years of fractures in boys and girls in Rogaland county, Norway, 1992-95, by age and treatment disposition.

or unorganized sports activities, excluding school injuries. Other events were all cases not represented in the above categories, for example, fractures occurring at home, in daycare centers, in shopping areas, on public or private playgrounds, as well as non-traffic transport injuries.

INCIDENCE

The size of the population enabled us to analyze incidence by age and sex in one year age intervals. Incidence was the number of fractures divided by the respective number of children years. We calculated age specific and sex specific incidence rates by treatment disposition, types of fracture, mechanism of occurrence, and category of event. Confidence intervals (CIs) for incidence rates were calculated to permit test for comparisons of person time incidence rates. ${ }^{8}$ Population census data were obtained from official data sources which, in Norway, are updated annually.

ESTIMATION OF DAYS OF ACTIVITY RESTRICTION Data on number of activity restriction days were available for a random sample of cases as a part of a larger study. ${ }^{9}{ }^{10}$ The sample includes 207 children treated at the Central Hospital in

Table 1 Annualised incidence of fractures per 10000 children by mechanism, category, and fracture site

\begin{tabular}{|c|c|c|c|c|}
\hline & \multicolumn{2}{|l|}{$0-6$ years } & \multicolumn{2}{|l|}{$7-12$ years } \\
\hline & $\begin{array}{l}\text { Boys (57 } 291 \\
\text { children years }\end{array}$ & $\begin{array}{l}\text { Girls (53 } 803 \\
\text { children years }\end{array}$ & $\begin{array}{l}\text { Boys ( } 41923 \\
\text { children years }\end{array}$ & $\begin{array}{l}\text { Girls ( } 40523 \\
\text { children years }\end{array}$ \\
\hline \multicolumn{5}{|l|}{ Mechanism } \\
\hline Fall & 72 & 60 & 131 & 118 \\
\hline Collision & 14 & 7 & 47 & 36 \\
\hline Other & 12 & 11 & 16 & 14 \\
\hline \multicolumn{5}{|l|}{ Category } \\
\hline Traffic & 6 & 6 & 23 & 17 \\
\hline School & 2 & 2 & 54 & 38 \\
\hline Sports & 6 & 3 & 45 & 39 \\
\hline Other & 83 & 65 & 73 & 74 \\
\hline \multicolumn{5}{|l|}{ Site } \\
\hline Arm & 52 & 47 & 135 & 125 \\
\hline Leg & 21 & 16 & 32 & 28 \\
\hline Other & 24 & 15 & 29 & 14 \\
\hline
\end{tabular}

^ Number of children years of follow up.
Rogaland. Parents were sent a questionnaire concerning the duration of the child's activity restriction. These questionnaires were sent at the beginning of 1994, between 1-2 years after the injury occurred. It was not possible to track the addresses of 15 children. Of 192 questionnaires sent, responses were received for 129 $(67 \%)$. For six children information about activity restriction was not provided, reducing the sample to 123 children.

The questionnaire asked about two types of activity restriction, complete or partial inability to perform usual leisure time activities. The total number of days of restricted activity for each child was calculated by weighting days with partial restriction by $50 \%$ and adding this weighted value to the days of complete restriction.

We calculated the proportion of children with any activity restriction, and the mean number of activity restricted days by location of fracture. We also calculated the incidence of fractures causing any activity restriction. Further, we calculated the number of activity restricted days per 10000 children in the population by multiplying the average number of activity restricted days by the incidence rate for each type of fracture.

\section{Results}

The study population had 2477 fractures, 128 per 10000 children years. Of these, 1378 (139 per 10000 children years) occurred in boys and 1099 in girls (117 per 10000 children years). The boy:girl incidence rate ratio was 1.2 (95\% CI 1.1 to 1.3 ).

Figure 1 shows the age specific incidence of fractures for boys and girls according to treatment disposition. The incidence rate increased approximately linearly with age in both girls and boys. The average annual increase in incidence was approximately 14 cases per 10000 children years. The incidence rate was consistently higher among boys than girls older than 2 years. The figure also shows the incidence of hospitalization due to fractures. Of the 2477 children with fractures, 485 $(20 \%)$ were hospitalized. Of these, 293 were boys and 192 were girls. The boy:girl incidence rate ratio was 1.45 (95\% CI 1.21 to 1.74 ). The hospitalization rate increased until the age of 7 for both boys and girls. Among children age 8 and older, the hospitalization rate remained stable and even declined slightly among boys.

Table 1 shows the annual incidence of fractures by mechanism, category of event, fractures site, and treatment disposition. The most common mechanism of injury was a fall, representing $71 \%$ of all cases. The incidence of fractures due to falls increased with age, and equally for both sexes. Collision caused $19 \%$ of all fractures and the incidence of these fractures also increased with age. All other mechanisms together caused $10 \%$ of the fractures and incidence of these exhibited little change with age. Fractures occurring in school represented $17 \%$ of the cases, sports (excluding school sports) $16 \%$, traffic $9 \%$, and other fractures, $58 \%$. Of the last group the majority 
Table 2 Activity restricted days among children by type of fracture

\begin{tabular}{|c|c|c|c|c|c|}
\hline & \multirow[b]{2}{*}{ No } & \multicolumn{2}{|c|}{$\begin{array}{l}\text { Proportion with } \\
\text { disability }\end{array}$} & \multicolumn{2}{|c|}{$\begin{array}{l}\text { Activity restricted } \\
\text { days }\end{array}$} \\
\hline & & $(\%)$ & $95 \% C I$ & Mean & $95 \% C I$ \\
\hline Arm & 85 & 72 & 61 to 81 & 14 & 8 to 20 \\
\hline Arm (excluding wrist/hand/finger) & 41 & 90 & 77 to 97 & 22 & 11 to 33 \\
\hline Wrist/hand/finger & 44 & 55 & 39 to 70 & 6 & 3 to 10 \\
\hline Leg & 19 & 84 & 60 to 97 & 26 & 7 to 45 \\
\hline Leg (excluding ankle/foot/toe) & 11 & 91 & 59 to 100 & 39 & 9 to 71 \\
\hline Ankle/foot/toe & 8 & 75 & 35 to 97 & 7 & 0 to 15 \\
\hline Other & 19 & 58 & 34 to 80 & 5 & 1 to 8 \\
\hline
\end{tabular}

occurred at home. Of the 235 traffic related fractures, $83 \%$ occurred among children riding a bicycle and $13 \%$ among pedestrians.

Arm fractures were the most common, representing $65 \%$ of all cases. Leg fractures were the second most common $(18 \%$ of all cases), and all other fractures, $16 \%$. The incidence of arm fractures increased linearly with age, in both boys and girls, by approximately 12 cases per 10000 children annually (not shown in table 1). The incidence of leg and other fractures increased until the age of 3 , and then remained constant.

The effect of fractures on daily activities expressed as the proportion of injured children experiencing any activity restriction and as the number of activity restricted days is shown in table 2 . The highest degree of activity restriction was associated with fractures of the leg, followed by fractures of the arm. The difference in number of activity restricted days among different types of fractures was statistically significant (Kruskal-Wallis analysis of variance, $\mathrm{p}<0.05)$.

Table 3 shows the incidence of fractures causing activity restriction, and number of days of activity restriction. Overall, $72 \%$ resulted in activity restriction. Due to differences in length of activity restriction among different types of fractures, the relative distribution of number of activity restriction days in the population differed from the relative distribution of the cases in the population. Leg fractures, although representing only $19 \%$ of the cases, accounted for $33 \%$ of all activity restricted days. Fractures sustained in traffic (including lone bicycle injuries) represented approximately one tenth of the cases and activity restricted days. School and sports injuries each represented approximately $15 \%$ of the cases and days of restricted activity. The majority of fractures and days of restricted activity were caused by "other fractures".

Table 3 Annualised incidence of fractures causing activity restriction per 10000 children aged 0-12 years and number of activity restricted days due to fracture per 10000 children aged 0-12 years by type of fracture and category of event

\begin{tabular}{llllll}
\hline & \multicolumn{2}{l}{ Incidence } & & \multicolumn{2}{c}{ Activity restricted days } \\
\cline { 2 - 3 } \cline { 5 - 6 } \cline { 5 - 6 } & Rate & $95 \%$ CI & & Per population & $95 \% C I$ \\
\hline Location & & & & \\
Arm & 60 & 51 to 67 & & 1179 & 692 to 1667 \\
Leg & 20 & 14 to 23 & & 615 & 174 to 1055 \\
Other & 12 & 7 to 17 & & 98 & 22 to 174 \\
Category & 8 & 6 to 10 & & 167 & 75 to 259 \\
Traffic & 15 & 12 to 18 & & 317 & 156 to 478 \\
School & 15 & 12 to 17 & & 307 & 145 to 469 \\
Sports & 53 & 42 to 62 & & 1102 & 511 to 1693 \\
Other & & & & & \\
\hline
\end{tabular}

\section{Discussion}

This study describes the occurrence of fractures in a large defined population of children. The incidence of fractures among children aged $0-12$ years increased with age and the majority of this increase was due to arm fractures. The injury categories showing this age dependent increase in incidence were school, sports, and bicycle related fractures.

We did not identify any significant difference in patterns of fracture occurrence between boys and girls. Boys and girls experienced a similar increase in incidence of fractures with age. Further, the relative distribution of fractures by type, mechanism, and category of injury did not differ. It is, however, important to emphasize that we analyzed only aggregate categories of fractures. More detailed analyses may reveal differences between boys and girls in patterns of injuries (for example types of sports).

The total incidence of fractures in our study population was 128 per 10000 children years. Few studies have reported the incidence of fracture among children. In one US study, the estimated incidence among children aged $0-19$ years was 268 per 10000 children years. ${ }^{1}$ In another US study the estimated incidence in the same age group was 390 per 10000 children years. ${ }^{3}$ These studies did not report age specific data that would allow for precise comparison with our rates. Differences in age, time of the study, or other factors could account for this difference in incidence. Little is known about the underlying sources of differences in childhood injury risks within and among countries. Studying these differences may help promote better understanding of underlying risk factors, thereby allowing the design of more effective preventive strategies.

This study shows that length of activity restriction due to fractures among children depends on the type of fracture. The longest per case activity restriction was associated with leg fractures, followed by arm fractures. Further segregation of arm and of leg fractures suggests that significant differences in activity restriction exist, for example, a common childhood fracture-fracture of the wrist-appears to be less disabling than fractures of long arm bones. Due to these differences, the incidence rate alone is a limited predictor of amount of activity restriction that will result from injuries to children. For example, fractures of the leg and the group of "other" fractures (head and trunk bones) had similar incidence rates, but the per population number of days of restricted activity was almost six times higher for fractures of the leg.

Days of restricted usual leisure time activities represent only one consequence of fractures in children. Others include, for example, long term disability, bed disability days, and loss of functional capacity. Unfortunately, there is little information about those consequences among children, although results from studies focusing on this have started to appear in the literature. ${ }^{11}$ 
The validity of our findings is dependent on the completeness of the registration, the accuracy of information included in the registry, and on the validity of the estimates of the days of activity restriction. The prospective injury registration system at the Central Hospital in Stavanger has operated since 1990 and is subject to continuous quality surveillance. The only cases missing from the registry are fractures that occurred and were treated outside the county. These cases represent a minor portion of all cases and, as such, are of little practical importance. Information included in the register is collected shortly after the injury (during the first visit to hospital) and is thus not affected by recall bias or response rate problems.

Our estimates of days of activity restriction are subject to several limitations. The sample used to estimate length of disability is relatively small, reducing precision of the estimates and preventing detailed segregation of cases by type of fracture and age of child. Length of activity restriction shows large individual variations, and a much larger sample than the one available is required if more precise estimates are to be derived. Estimates of activity restricted days were based on parent reported information obtained 1-2 years after the injury and thus may be subject to recall bias. Our estimates appear to have adequate face validity. Fractures that one would expect to cause more activity restriction, based upon clinical experience, such as fractures of long bones, were associated with longer activity restriction. Further, our findings were consistent with other findings reported previously. ${ }^{6}$

Our calculation of disability days is based on a weighted sum of days of complete and partial disability. The validity of this estimate can be questioned. We performed sensitivity analysis by varying the weighting factor from 0.3 to 0.7 and the results did not significantly change.

Our finding of a difference in length of activity restriction among fractures of different types is likely to be generalizable. The per population estimates of incidence and the number of fracture related days of restricted activity are, probably, setting specific. Our setting can be considered generally representative of Norway, and possibly of Scandinavia. To what extent these findings can be generalized to other countries and regions is less certain.

\section{Implications for prevention}

The findings of our study have implications for setting priorities in childhood injury prevention. The strong age gradient suggests that greater attention should be paid to injury risk among older children, particularly sports, school, and bicycle related injuries. Prevention efforts targeting these sources of fractures should not aim at reducing exposure, because participation in these activities is socially desirable. Rather, preventive measures should aim to reduce the level of risk, for example encouraging children to use safety equipment.

1 Walsh SSM, Jarvis SN, Towner, et al. Annual incidence of unintentional injury among 54000 children. Inj Prev 1996;2:16-20.

2 Gallagher SS, Finison K, Guyer B, et al. The incidence of njuries among 87000 Massachusetts children and adolescents: results of the 1980-81 statewide childhood injury prevention program surveillance system. Am f Public Health 1984;74:1340-7.

3 Rivara FP, Calonge N, Thompson RS. Population-based study of unintentional injury incidence and impact during childhood. Am f Public Health 1989;79:990-4.

4 Scheidt PC, Harel Y, Trumble AC, et al. The epidemiology Scheidt PC, Hare Y, Trumble AC, et al. The epidemiology
of nonfatal injuries among US children and youth. $A m \mathcal{F}$ Public Health 1995;85:932-8.

5 Walsh SS, Jarvis SN. Measuring the frequency of "severe" accidental injury in childhood. I Epidemiol Community Health 1992;46:26-32.

6 Rivara FP, Thompson RS, Thompson DC, et al. Injuries to children and adolescents: impact on physical health. Pediatrics 1991;88:783-8.

7 Barker M, Power C, Roberts I. Injuries and the risk of disability in teenagers and young adults. Arch Dis Child 1996;75:156-8.

8 Rothman KJ. Modern epidemiology. Boston: Little, Brown, 1986.

9 Kopjar B, Wickizer T. Cycling to school-a significant health risk? Inj Prev 1995;1:238-41.

10 Kopjar B, Wickizer T. How safe are day-care centers? Daycare versus home injuries among children in Norway. care versus home in

11 Gofin R, Adler B. A seven item scale for the assessment of disabilities after child and adolescent injuries. Inj Prev 1997;3:120-3 